\title{
Determinação do fator de correção de frutas e hortaliças de uma unidade de alimentação e nutrição fornecedora de refeições transportadas em Fortaleza - CE
}

Correction factor determination of fruits and vegetables from a food and nutrition unit providing

transported meals in Fortaleza - CE

Determinación del factor de corrección para frutas y verduras en una unidad de alimentación y nutrición proveedora de alimentos transportados en Fortaleza - CE

Leandro Santiago Hori ORCID: https://orcid.org/0000-0002-6692-6768 Universidade Estadual do Ceará, Brasil

E-mail: leandroshori@gmail.com

Denise Regina de Oliveira Brasil ORCID: https://orcid.org/0000-0002-5447-7360 ISM Gomes de Mattos EIRELI, Brasil E-mail: denise@ism.ind.br

Wilma Stella Giffoni Vieira

ORCID: https://orcid.org/0000-0002-9787-1157 ISM Gomes de Mattos EIRELI, Brasil E-mail: wilma.giffoni@ism.ind.br

Marilia de Carvalho Gonçalves ORCID: https://orcid.org/0000-0001-9368-6547 Universidade Estadual do Ceará, Brasil E-mail: marimariliag@gmail.com

Amauri Barbosa da Silva Junior ORCID: https://orcid.org/0000-0002-1647-2331 Universidade Estadual do Ceará, Brasil E-mail: amauribdsj@gmail.com

Renata Carmo de Assis

ORCID: https://orcid.org/0000-0003-2258-3844 Universidade Estadual do Ceará, Brasil E-mail: renata.carmo@uece.br

Nayranne Hivina Carvalho Tavares ORCID: https://orcid.org/0000-0002-3365-9552 Universidade Estadual do Ceará, Brasil E-mail: nayranne.hivina@uece.br

Clarice Maria Araújo Chagas Vergara ORCID: https://orcid.org/0000-0003-1709-9951 Universidade Estadual do Ceará, Brasil E-mail: clarice.araujo@uece.br

\begin{abstract}
Resumo
Frutas e hortaliças constituem alimentos importantes nas refeições de populações para um estilo de vida saudável e equilibrado. São caracterizadas por perdas e desperdícios ao longo da cadeia produtiva até o consumo final. O tipo de alimento e o seu grau de maturação, juntamente da técnica empregada para a remoção de partes não comestíveis são fatores que causam impactos no Fator de Correção (FC). Assim, o objetivo desse estudo foi determinar o FC de frutas e hortaliças de uma Unidade de Alimentação e Nutrição (UAN) fornecedora de refeições transportadas em Fortaleza Ceará. Trata-se de um estudo descritivo, transversal, de caráter quantitativo, realizado no período de dezembro de 2020 a janeiro de 2021. A partir da divisão do Peso Bruto pelo Peso Líquido, foram determinados os FC dos alimentos, estabelecendo a média aritmética após coleta em triplicata. Comparou-se os valores médios obtidos com os da literatura, observando-se os aspectos do alimento e o tipo de corte. Assim, verificou-se que, das hortaliças, o FC médio da abobrinha estava acima dos estudos comparados. Em relação às frutas, apenas o melão apresentou valores superiores aos encontrados na literatura. Dessa forma, os FC de frutas e hortaliças foram considerados satisfatórios quando comparados aos encontrados na literatura, levando em consideração o nível de tecnologia e a mão-de-obra disponível no serviço. Contudo, ressalta-se a importância dos treinamentos de manipuladores quanto às boas práticas com enfoque no desperdício de alimentos, assim como acompanhamento diário de colaboradores durante o pré-preparo desses alimentos para a melhoria da qualidade da UAN.
\end{abstract}

Palavras-chave: Frutas; Hortaliças; Desperdício de alimentos. 


\begin{abstract}
Fruits and vegetables are important foods in people's meals for a healthy and balanced lifestyle. They are characterized by losses and waste along the production chain until final consumption. The type of food and its degree of maturation, together with the technique used to remove inedible parts, are factors that impact the Correction Factor (FC). Thus, the objective of this study was to determine the CF of fruits and vegetables of a Food and Nutrition Unit (UAN) that provides transported meals in Fortaleza - Ceará. This is a descriptive, cross-sectional, quantitative study, carried out from December 2020 to January 2021. From the division of Gross Weight by Net Weight, the FC of the food was determined, establishing the arithmetic mean after collection in triplicate. The mean values obtained were compared with those in the literature, observing the aspects of the food and the type of cut. Thus, it was found that, for vegetables, the mean $\mathrm{CF}$ of zucchini was above the compared studies. In relation to fruits, only melon presented values higher than those found in the literature. Thus, the CF of fruits and vegetables were considered satisfactory when compared to those found in the literature, taking into account the level of technology and labor available in the service. However, it emphasizes the importance of training handlers on good practices with a focus on food waste, as well as daily monitoring of employees during the pre-preparation of these foods to improve the quality of the UAN.
\end{abstract}

Keywords: Fruits; Vegetables; Food losses.

\title{
Resumen
}

Las frutas y verduras son alimentos importantes en las comidas de las personas para un estilo de vida saludable y equilibrado. Se caracterizan por pérdidas y desperdicios a lo largo de la cadena de producción hasta el consumo final. El tipo de alimento y su grado de maduración, junto con la técnica empleada para eliminar las partes no comestibles, son factores que inciden en el Factor de Corrección (FC). Así, el objetivo de este estudio fue determinar la FC de frutas y verduras de una Unidad de Alimentación y Nutrición (UAN) que provee alimentos transportados en Fortaleza - Ceará. Se trata de un estudio descriptivo, transversal, cuantitativo, realizado de diciembre de 2020 a enero de 2021. A partir de la división del Peso Bruto por el Peso Neto se determinó la FC del alimento, estableciendo la media aritmética después de la recolección por triplicado. Los valores medios obtenidos se compararon con los de la literatura, observando los aspectos de la comida y el tipo de corte. Así, se encontró que, para las verduras, la FC media del calabacín era más alta que en los estudios comparativos. En relación a los frutos, solo el melón presentó valores superiores a los encontrados en la literatura. Así, las FC de frutas y hortalizas se consideraron satisfactorias al compararlas con las encontradas en la literatura, tomando en cuenta el nivel de tecnología y mano de obra disponible en el servicio. Sin embargo, enfatiza la importancia de capacitar a los manipuladores en buenas prácticas con enfoque en el desperdicio de alimentos, así como el seguimiento diario de los empleados durante la pre-preparación de estos alimentos para mejorar la calidad de la UAN. Palavras clave: Frutas; Verduras; Desechos alimentarios.

\section{Introdução}

Unidades de Alimentação e Nutrição (UANs) fornecedoras de refeições transportadas caracterizam-se pela descentralização em relação a produção da refeição com o local de distribuição. Assim como na forma centralizada, esses serviços consistem em um conjunto de setores nos quais se operam a produção de alimentos para coletividades, constituindo, dessa forma, um serviço sistemático, com o qual se objetiva o cuidado nutricional de indivíduos através de refeições equilibradas do ponto de vista não só dietético, mas com aspectos seguros à ingestão alimentar. (Souza; Leite; Costa, 2016; Silva Junior, 2020). No ano de 2020, estima-se que o segmento de refeições coletivas tenha produzido, aproximadamente, 14,7 milhões de refeições por dia por empresas prestadoras de serviços (ABERC, 2015).

Frutas e hortaliças constituem alimentos perecíveis importantes nas refeições de populações para um estilo de vida saudável e equilibrado, visto que são fornecedoras de fibras, vitaminas e minerais. São caracterizadas por terem perdas e desperdícios ao longo da cadeia produtiva até o consumo final, sendo importante investir em tecnologia e inovação no que diz respeito a esses aspectos (FAO, 2021).

O tipo de alimento e o seu grau de maturação, juntamente com a técnica empregada para a remoção de partes não comestíveis, são fatores que causam impactos no FC, o qual relaciona o Peso Bruto e o Peso Líquido para calcular a perda dos alimentos durante o pré-preparo (Ornellas, 2007). Consequentemente, tais aspectos podem implicar no aumento de despesas de serviços de alimentação e nutrição para a obtenção desses insumos (Teixeira et al., 2007; Domene, 2018).

O desperdício na produção de refeições em UANs é variado e pode ter diversos fatores para além do alimento, como falta de capacitações periódicas de manipuladores, falta de processos padronizados e falta de monitoramento das ações 
empreendidas por colaboradores (de Andrade Silvério \& Oltramari, 2014). O treinamento de manipuladores de alimentos que atuam no beneficiamento de hortifrutis, a ordem de realização dos procedimentos, bem como o nível tecnológico disponível nos serviços de alimentação para beneficiamento, armazenamento e monitoramento influenciam na geração de resíduos sólidos nas etapas de pré-preparo desses alimentos, caso não sejam aproveitadas partes como talos, cascas e sementes (Santos Junior, 2013; Azevedo \& Costa, 2017).

A redução do desperdício de alimentos ao longo da cadeia de produção de refeições é de grande importância para o controle de custos. Nesse sentido, a utilização de novas tecnologias na cadeia de produção de refeições aprimora a sua atividade fim, reduzindo gastos com matéria-prima (Oliveira \& Silva, 2016).

Dentre os desafios do nutricionista em UANs, destacam-se a implantação e o monitoramento de ações de produção sustentável, que possam impactar na minimização de desperdícios, sobras e restos (CFN, 2018). Assim, é relevante que nos serviços de alimentação sejam estabelecidos fatores de correção continuamente, a fim de melhorar o controle de qualidade, considerando a importância destes indicadores à diminuição de perdas de alimentos (Goes; Valduga \& Soares, 2013).

Tendo em vista a melhoria dos processos no beneficiamento de hortifrutis, o objetivo deste trabalho foi determinar o Fator de Correção de frutas e hortaliças em uma Unidade de Alimentação e Nutrição fornecedora de alimentação transportada de Fortaleza - Ceará.

\section{Metodologia}

Trata-se de um estudo descritivo, transversal, de caráter quantitativo, realizado no período de dezembro de 2020 a janeiro de 2021 no setor de pré-preparo de frutas, legumes e verduras de uma Unidade de Alimentação e Nutrição fornecedora de refeições transportadas de Fortaleza - CE. A UAN fornece refeições para diferentes instituições, e os per capitas de frutas e hortaliças variam de acordo com as especificações dos contratos

Assim, a coleta dos dados ocorreu conforme a incidência dos hortifrutis no cardápio no período analisado, e foi realizada por estagiários curriculares do curso de nutrição. Todos os alimentos utilizados no estudo diferentes de frutas foram categorizados como hortaliças, conforme a definição e classificação de Ornellas (2007). Assim, os alimentos utilizados na pesquisa foram divididos em dois grupos: 1. Hortaliças (abóbora leite, abobrinha, alface crespa, cebola, cebolinha, coentro, cenoura, chuchu, batata-doce, batata inglesa, beterraba, pimentão verde e vagem) e 2. Frutas (abacaxi, mamão, melancia e melão).

Para a determinação do Fator de Correção (FC), foram determinados o Peso Bruto (PB) e o Peso Líquido (PL) de cada alimento e calculado através da fórmula: $\mathrm{FC}=\mathrm{PB} / \mathrm{PL}$. As amostras foram coletadas em triplicata, por porções ou por unidade, as quais ocorreram em dias alternados. Ao final, foram calculadas as médias aritméticas dos três valores de FC obtidos de cada alimento. As aferições de PB e PL foram realizadas em balança digital de plataforma Balmak® modelo BK-300 calibrada, com precisão de 100g.

Para a obtenção de Peso Líquido (PL) foram realizadas as remoções de talos, cascas, sementes, folhas deterioradas ou velhas, partes não comestíveis, os quais foram feitos, manualmente, por manipuladores treinados e/ou descascador industrial Incalfer® modelo P60 / 2013 antes da etapa de higienização e desinfecção dos hortifrutis. Os instrumentos utilizados pelos manipuladores foram: faca, colher, descascador manual e descascador industrial, este último, para os legumes que não necessitam da etapa de desinfecção.

Utilizou-se o programa Microsoft Excel® 2013 para realizar o cálculo de FC e a média da triplicata de cada alimento. Após isso, os FC médios foram comparados com os da literatura, adotando-se Ornellas (2007) como referência primária e Silva, de Jesus e Soares (2016) como referência secundária, observando os aspectos do alimento e o tipo de corte empregado para a remoção das aparas. 
Ressalta-se que o presente estudo se encontra com Parecer Consubstanciado do Comitê de Ética em Pesquisa da Universidade Estadual do Ceará aprovado, através do número 3.285.724.

\section{Resultados e Discussão}

Com relação a qualidade da matéria-prima, todos os hortifrutis estavam em bom estado de conservação, conforme padrão exigido pela UAN, armazenados em câmaras de refrigeração sob temperatura controlada, sendo uma para hortaliças que necessitavam de refrigeração e outra para as frutas. As hortaliças que não necessitavam de refrigeração, como a abóbora leite, eram armazenadas à temperatura ambiente. Conforme preconiza Silva Junior (2020), os alimentos estavam dispostos e organizados em monoblocos, sobre pallets, de forma a preservar a integridade dos alimentos, a fim de se obter maior controle dos alimentos que entram e saem do setor de armazenamento.

As hortaliças eram manipuladas para a produção de saladas cozidas e cruas, e para a fabricação de temperos das preparações. As frutas, por sua vez, eram beneficiadas para serem utilizadas em sobremesas, e em saladas cruas. Quanto ao recebimento dessas mercadorias, a ocorrência era de três vezes por semana, em local próprio para esse fim, para suas devidas conferências e avaliações, respeitando os Procedimentos Operacionais Padronizados (POPs) de recebimento e de armazenamento dessas matérias-primas específicas.

A Tabela 1 traz os valores de fator de correção encontrados neste estudo. Dentre as hortaliças avaliadas, o FC médio da abobrinha, da alface e do coentro estavam acima do estabelecido por Ornellas (2007), que foram determinadas em situações de laboratório, diferentemente do presente trabalho. Contudo no estudo de Silva, Jesus e Soares (2016), os quais estabeleceram Fator de Correção de frutas e hortaliças em UANs de Salvador - BA, os valores para estes alimentos diferiram: encontrou-se um valor maior para a alface crespa $(1,65-2,08)$ em relação ao determinado neste estudo $(1,62)$; enquanto foi encontrado um valor de 1,35 para o coentro, que está dentro do intervalo estabelecido pelas pesquisadoras $(1,18-1,96)$. Com relação aos folhosos, o estudo das pesquisadoras verificou que a qualidade, bem como seu adequado armazenamento, além da etapa de beneficiamento, que era realizada pelos mesmos manipuladores, foram fatores relacionados a menores perdas. 
Tabela 1. Fator de Correção Médio de Hortaliças de UAN de grande porte fornecedora de refeições transportadas em Fortaleza - CE.

\begin{tabular}{|c|c|c|c|c|c|c|c|}
\hline Alimento & Instrumento & FC 1 & FC 2 & FC 3 & $\begin{array}{l}\text { FC } \\
\text { médio }\end{array}$ & $\begin{array}{l}\text { FC Ornellas } \\
(2007)\end{array}$ & $\begin{array}{l}\text { FC médio Silva, } \\
\text { Jesus, Soares } \\
(2016)\end{array}$ \\
\hline $\begin{array}{l}\text { Abóbora } \\
\text { Leite }^{1}\end{array}$ & $\mathrm{DM}, \mathrm{FeC}$ & 1,36 & 1,32 & 1,18 & 1,28 & $1,15-1,64$ & - \\
\hline $\begin{array}{l}\text { Abóbora } \\
\text { Leite }^{2}\end{array}$ & $\mathrm{FeC}$ & 1,07 & 1,15 & 1,08 & 1,10 & $1,15-1,64$ & - \\
\hline Abobrinha $^{1}$ & DM, F e C & 1,62 & 1,60 & 1,70 & 1,64 & $1,33-1,38$ & \\
\hline & $\mathrm{F}$ & 1,47 & 1,48 & 1,90 & 1,62 & $1,09-1,33$ & $1,65-2,08$ \\
\hline Cebola & DI e F & 1,32 & 1,44 & 1,28 & 1,35 & $1,03-2,44$ & $1,19-1,48$ \\
\hline Cebolinha & $\mathrm{F}$ & 1,16 & 1,09 & 1,12 & 1,12 & - & $1,05-1,39$ \\
\hline Coentro & $\mathrm{F}$ & 1,41 & 1,28 & 1,37 & 1,35 & 1,10 & $1,18-1,96$ \\
\hline Cenoura & DI e F & 1,05 & 1,03 & 1,08 & 1,05 & 1,17 & $1,06-1,25$ \\
\hline Chuchu $^{1}$ & $\mathrm{DM}, \mathrm{F}$ e C & 1,44 & 1,41 & 1,44 & 1,43 & 1,47 & $1,16-1,17$ \\
\hline Batata-Doce & DI & 1,02 & 1,02 & 1,07 & 1,04 & $1,13-1,33$ & - \\
\hline $\begin{array}{l}\text { Batata } \\
\text { Inglesa }\end{array}$ & DI & 1,01 & 1,02 & 1,04 & 1,02 & 1,06 & $1,01-1,07$ \\
\hline Beterraba & DI e F & 1,13 & 1,13 & 1,04 & 1,10 & $1,61-1,88$ & - \\
\hline $\begin{array}{l}\text { Pimentão } \\
\text { Verde }\end{array}$ & $\mathrm{F}$ & 1,03 & 1,11 & 1,18 & 1,11 & 1,26 & 1,25 \\
\hline Vagem & $\mathrm{F}$ & 1,10 & 1,30 & 1,15 & 1,18 & 1,41 & $1,16-1,32$ \\
\hline
\end{tabular}

Legenda: ${ }^{1}$ Retiradas cascas e sementes; ${ }^{2}$ Retiradas sementes; ${ }^{3}$ Retiradas cascas; DM: Descascador Manual. DI: Descascador Industrial. F: Faca. FC: Fator de Correção. Fonte: Autores (2021).

Para a cebolinha, o fator estabelecido foi de 1,12, enquanto Silva, Jesus e Soares (2016) encontraram um intervalo de 1,09 - 1,39. Estudos de Silva, das Chagas Moura e Bezerra (2020) em UAN de Teresina - PI, encontraram o valor de 2,1 para a cebolinha, contudo, no estudo em questão, verificou-se uma retirada excessiva devido aos danos físicos e mecânicos, assim como a má qualidade da matéria-prima, os quais foram verificados durante a etapa de recebimento de mercadorias. Além disso, as autoras ressaltam a questão da falta de treinamento e da utilização de utensílios inadequados como fatores que acarretaram o aumento do FC em questão.

De modo geral, dentre os fatores associados aos desperdícios durante as etapas de pré-preparo de hortaliças e frutas na UAN do estudo, observados durante a coleta de dados, pode-se destacar que o instrumento utilizado para a remoção de partes não comestíveis dos alimentos, a técnica utilizada pelo manipulador e o estado de maturação dos alimentos foram os principais fatores relacionados às perdas.

No que diz respeito ao instrumento, verifica-se que alimentos que passaram exclusivamente pelo descascador industrial tiveram menores perdas em relação aos dados de Ornellas (2007), como é o caso da batata-doce e da batata inglesa com valores de 1,04 e 1,02 respectivamente. Comparado aos valores obtidos por Silva, Jesus e Soares (2016), o valor do FC da batata inglesa determinado $(1,02)$ encontrou-se dentro do intervalo encontrado pelas autoras $(1,01-1,07)$, contudo, as pesquisadoras utilizaram descascador manual e descascador elétrico para a retirada das partes não comestíveis.

A batata-doce, com fator de 1,04, ficou abaixo do valor estabelecido por Ornellas $(1,13-1,33)$, o que pode ser justificado pelo fato do alimento ter sido exclusivamente beneficiado pelo descascador industrial. Os estudos de Oliveira et al. (2020), em UAN comercial, demonstram os valores de 1,13 - 1,31 para o alimento em questão, mas não explanaram os métodos de remoção das aparas.

A cenoura, com fator de 1,05, passa por um processo de pré-beneficiamento, onde são removidos resquícios do caule e do ápice. Feito isso, o alimento é beneficiado no descascador industrial e depois recolhido para ser cortado conforme a 
preparação. Diferentemente, a cebola, com FC de 1,35, passa pelo processo inverso: primeiramente, elas são colocadas no descascador industrial, após isso, são recolhidas e retiradas as cascas remanescentes e, por fim, são removidos os talos e as raízes. Busato e Ferigollo (2018) ressaltam que o estabelecimento de rotinas nos processos de serviços de alimentação pode contribuir para a redução de perdas de alimentos.

Estudos de Araújo e Carvalho (2015), em UAN de Goiânia - GO, encontraram o valor de 1,2 para o chuchu, contudo, deixam claro que esse alimento ainda permaneceu com casca, sendo retiradas somente as sementes e pontas não comestíveis, o que indica que, de acordo com a finalidade da preparação, o FC pode ser maior ou menor. Outrossim, na presente pesquisa da UAN cearense, para a abóbora, existiam dois tipos de beneficiamentos: um em que permanecia a casca, mas sem sementes $(1,10)$; e outra sem cascas e sem sementes $(1,28)$, os quais são utilizados para preparações e clientelas diferentes. Ornellas (2007) adota o intervalo de 1,15 a 1,64, entretanto, não especifica o tipo de abóbora utilizado em seu estudo.

Além disso, verificou-se que, para determinadas preparações, alimentos, como o chuchu e a abobrinha, eram beneficiados de forma a estarem totalmente livres de cascas e sementes. Com isso, o tempo para realizar o pré-preparo foi maior, devido ao volume dos alimentos e pelo tipo de instrumento utilizado, que foram descascador manual, faca e colher. Conforme Silva, de Jesus e Soares (2016), o estresse do ambiente de trabalho, pode influenciar em maiores perdas. Igualmente, o treinamento periódico em boas práticas se faz importante nesse contexto de redução de desperdícios, sendo fundamental o acompanhamento e monitoramento diário da equipe de manipuladores (dos Reis Santos, 2019).

Nesse sentido, otimizar os processos de beneficiamento de hortifrutis, a fim de diminuir esses entraves pode ser uma questão de saúde ocupacional, visto que a manipulação dos alimentos realizada em pé, manualmente, através de movimentos repetitivos, pode acarretar Lesão por Esforço Repetitivo (LER) (de Souza \& Silveira, 2015).

A institucionalização de mais de um indicador de FC para o mesmo alimento, mas para preparações diferentes pode ser importante, quando se diz respeito à redução de custos e à melhora dos processos de qualidade em serviços de alimentação. Segundo estudo de Soares et al. (2011) acerca da quantificação e análise do custo da sobra limpa em UAN de uma empresa de grande porte, destaca-se a importância do planejamento de cardápios e da elaboração adequada das refeições para a melhoria dos processos produtivos e redução do desperdício.

Com relação às frutas, observou-se que o abacaxi, o mamão, a melancia e o melão são produtos que têm maior incidência na unidade por serem os mais utilizados na fabricação das sobremesas e das saladas cruas (Tabela 2). Os manipuladores que atuam nesse setor possuem experiência no beneficiamento de frutas e, são treinados e monitorados pelo nutricionista do setor. Tais aspectos podem levar ao aperfeiçoamento da técnica e, consequentemente, à redução do desperdício e dos valores do FC (Teixeira et al., 2017).

Tabela 2. Fator de Correção Médio de Frutas de UAN de grande porte fornecedora de refeições transportadas em Fortaleza CE.

\begin{tabular}{cccccccc}
\hline Alimento & Instrumento & FC 1 & FC 2 & FC 3 & FC médio & $\begin{array}{c}\text { FC Ornellas } \\
(\mathbf{2 0 0 7 )}\end{array}$ & $\begin{array}{c}\text { FC médio } \\
\text { Silva, Jesus, } \\
\text { Soares (2016) }\end{array}$ \\
\hline Abacaxi & F & 1,73 & 1,83 & 1,62 & 1,73 & 1,89 & $2,05-2,17$ \\
Mamão $^{1}$ & F e C & 1,46 & 1,77 & 1,51 & 1,58 & $1,47-1,79$ & $1,46-1,47$ \\
Melancia $^{3}$ & F & 1,38 & 1,46 & 1,32 & 1,39 & 2,17 & $1,44-1,50$ \\
Melão $^{1}$ & F e C & 1,64 & 1,60 & 1,65 & 1,63 & 1,04 & $1,45-1,53$ \\
\hline
\end{tabular}

Legenda: ${ }^{1}$ Retiradas cascas e sementes; ${ }^{2}$ Retiradas sementes; ${ }^{3}$ Retiradas cascas; F: Faca; C: Colher; FC: Fator de Correção. Fonte: Autores. (2021). 
No que diz respeito ao Fator de Correção do abacaxi, observou-se que o valor médio encontrado $(1,73)$ foi consideravelmente menor do que o valor apresentado por Ornellas $(2007)$ - $(1,89)$ e por Silva, de Jesus e Soares $(2016)$ - $(2,05$ -2,17). Quando comparado aos resultados obtidos no estudo de Canuto et al. (2019), que foi realizado por um período de oito meses com o intuito de relacionar a influência da safra dos hortifrútis no fator de correção, observou-se que o presente estudo apresenta um valor dentro do intervalo encontrado $(1,44$ - 2,95).

Isso pode ocorrer devido ao fato de que, no local onde o presente estudo foi realizado, os manipuladores retiraram as aparas com cuidado, a fim de evitar o desperdício dos alimentos, visto que é uma unidade que produz elevadas quantidades de refeições, e o impacto dos desperdícios nos custos da unidade seria bastante significativo. Enquanto isso, o estudo de Canuto et al. (2019) relata que seus resultados com FC elevados podem ter ocorrido por erros na manipulação dos hortifrutis e pela má conservação dos alimentos.

O presente estudo também avaliou o FC do mamão e obteve a média de 1,58, valor que se encaixa dentro dos padrões estabelecidos por Ornellas (2007), ligeiramente inferior quando comparado ao valor encontrado por Lacerda et al. (2014) em sua pesquisa (1,69), mas superior dos valores encontrados por Silva, de Jesus e Soares (2016), que foi de 1,46 - 1,47. O estudo de Silva, de Jesus e Soares (2016) não deixa evidente se eram retiradas as cascas do mamão, ao contrário do presente estudo, no qual foram retiradas cascas e sementes.

A melancia, de acordo com os resultados obtidos no presente trabalho, teve um fator de correção menor do que o encontrado na literatura, como mostra a Tabela 2. Isso pode ser em decorrência de que, no presente trabalho, não foram retiradas as sementes, apenas as cascas. Já o melão (1,63), ao contrário da melancia, apresentou o FC médio superior tanto para Ornellas (2007), quanto para Silva, de Jesus e Soares (2016), o que pode ser justificado tanto pela retirada das cascas e das sementes, reduzindo mais o peso líquido do alimento.

Assim, observou-se a influência do grau de maturação da fruta, pois tanto nos mamões quanto nos melões maduros, as retiradas se tornavam mais facilitadas, promovendo um corte acima do ideal, o que pode ter acarretado maiores perdas junto às cascas. De acordo com Lima (2016), as perdas de partes comestíveis de frutas no pós-colheita podem ocorrer devido ao amadurecimento e a senescência, os quais são fatores que devem estar de acordo com o armazenamento adequado e seu manuseio correto.

Enfatiza-se que, durante a coleta de dados, os manipuladores que realizaram o pré-preparo dos hortifrutis não receberam orientações adicionais quanto ao desperdício dos alimentos ou qualquer informação que pudesse influenciar na obtenção dos dados obtidos. Ressalta-se, ainda, que foram tomados cuidados referentes às Boas Práticas de Manipulação, como o uso de luvas descartáveis, de utensílios adequados e de cubas higienizadas, além de que foram obedecidas as Instruções de Trabalho do local.

O fato de que a UAN atendia a diferentes clientes, as quais possuíam especificações de cardápio diferentes, embora as preparações fossem as mesmas, pode ter impactado em diferentes perdas. Com isso, o presente estudo amplia as pesquisas na área de alimentação coletiva, considerando a variedade de hortaliças e frutas do estudo e seus respectivos fatores de correção, sendo importante novas investigações deste índice nos serviços de alimentação.

\section{Conclusões}

Os fatores de correção de frutas e hortaliças determinados neste estudo foram considerados satisfatórios, levando-se em consideração o nível de tecnologia empregado e a mão-de-obra disponível no serviço, em comparação com os valores encontrados na literatura. Contudo ressalta-se a importância do treinamento de manipuladores quanto às boas práticas com enfoque no desperdício de alimentos, assim como o acompanhamento diário desses colaboradores durante o pré-preparo de frutas e hortaliças para a melhoria da qualidade do processo de beneficiamento destes alimentos na UAN. 
Diante do exposto, devido as frutas e hortaliças serem utilizadas em grandes quantidades e em diferentes preparações, é necessário que os fatores de correção dos gêneros sejam constantemente investigados, para que assim sejam evitados desperdícios de alimentos.

\section{Referências}

Araújo, E. L. M., Martins, A. C., \& Carvalho, S. (2015). Sustentabilidade e geração de resíduos em uma unidade de alimentação e nutrição da cidade de GoiâniaGO. Demetra: alimentação, nutrição \& saúde, 10(4), 775-796.

ABERC, Associação Brasileira das Empresas de Refeições Coletivas. (2015). Manual ABERC de Práticas de Elaboração e Serviço de Refeições para Coletividades 2015. (11a ed.) São Paulo. http://www.aberc.com.br/mercadoreal.asp?IDMenu=21

Azevedo, A. M. F., \& Costa, A. M. M. (2017). Determinação e Avaliação do Índice de Parte Comestível de vegetais em uma Unidade de Alimentação e Nutrição de Fortaleza, Ceará. Rev. Simbio-Logias, 9 (12), 65-82.

Busato, M. A., \& Ferigollo, M. C. (2018). Desperdício de alimentos em unidades de alimentação e nutrição: uma revisão integrativa da literatura. Holos, 1, 91102.

Canuto, I. G., dos Santos Viana, L. S., da Rocha, B. R. S., \& de Carvalho, I. M. M. (2019). Estimativa do fator de correção em função da safra das principais hortaliças e frutas utilizadas no restaurante universitário da universidade federal de Sergipe (RESUN/UFS)/ Estimation of the correction factor according to the harvest of the main vegetables and fruits used at the University Restaurant of the Federal University of Sergipe (RESUN/UFS). Brazilian Journal of Development, 5(10), 19827-19840.

CFN, Conselho Federal de Nutricionistas. (2018). Resolução CFN n ${ }^{\circ}$ 600, 25 de fevereiro de 2018. Dispõe sobre as atividades dos nutricionistas. Diário Oficial da União. https://www.cfn.org.br/wp-content/uploads/resolucoes/Res_600_2018.htm

de Andrade Silvério, G., \& Oltramari, K. (2014). Desperdício de alimentos em Unidades de Alimentação e Nutrição brasileiras Food waste in Brazilian Units Food and Nutrition. AMBIÊNCIA, 10(1), 125-133.

de Oliveira, K. P., Pontes, C. R., Maia, S. M. P. C., Vieira, J. M. M., \& Pereira, C. P. (2020). Intervenção para redução de custos em um restaurante comercial em Fortaleza, Ceará. Research, Society and Development, 9(7), e925974472-e925974472.

de Souza, R. C., \& Silveira, F. S. A. (2015). Análise ergonômica do trabalho (aet) em uma unidade de produtora de refeição na cidade de Viçosa, MG. ANAIS SIMPAC, 3(1).

Domene, S. M. A. (2018). Técnica Dietética: Teorias e Aplicações. (2a ed.), Guanabara Koogan.

dos Reis Santos, J. A. (2019). Mapeamento dos processos de boas práticas na manipulação de alimentos com ênfase na redução de custos, qualidade e segurança alimentar em um restaurante de Foz do Iguaçu/PR. Biblioteca Digital de TCC-UniAmérica, 1-13.

Food And Agriculture Organization Of The United Nations, FAO. (2021). Fruit and vegetables - your dietary essentials. The International Year of Fruits and Vegetables, background paper. Rome.

Goes, V. F., Valduga, L., \& Soares, B. M. (2013). Determinação e Avaliação do Fator de Correção de Hortaliças em uma Unidade de Alimentação e Nutrição de Guarapuava-PR. Journal of Health Sciences.

Lacerda, L. L., Saraiva, B. C. A., Silva, Y. L., \& Monteiro, M. R. P. (2014). Avaliação do desperdício de hortifrútis em Unidades Produtoras de Refeição. DEMETRA: Alimentação, Nutrição \& Saúde, 9(3), 823-831.

Lima, J. A. D. (2016). Métodos para conservação de frutas e hortaliças.

Oliveira, T. C., \& Silva, D. A. (2016). Administração de Unidades Produtoras de Refeiçães: Desafios e perspectivas. Rubio.

Ornellas, L. H. (2007). Técnica Dietética: Seleção e preparo de alimentos. (8a ed.), Atheneu.

Santos Junior, C. J. (2013). Manual de Segurança Alimentar: Boas Práticas para os serviços de manipulação. (2a ed.), Rubio.

Silva Junior, E. A. (2020). Manual de Controle Higiênico-Sanitário em Serviços de Alimentação. (7a ed.), Varela.

Silva, C. S., de Jesus, J. C., \& Soares, L. S. (2016). Fator de Correção de Frutas e hortaliças em unidades de alimentação e nutrição de Salvador-Ba. Higiene Alimentar, 30(262/263).

Silva, N. B., das Chagas Moura, V. M., \& Bezerra, K. C. B. (2020). Avaliação do fator de correção de hortifrútis em uma unidade de alimentação e nutrição de Teresina-PI. Brazilian Journal of Development, 6(3), 13138-13146.

Soares, I. C. C., Silva, E. R. D., Priore, S. E., Ribeiro, R. D. C. L., Pereira, M. M. L. D. S., \& Pinheiro-Sant'Ana, H. M. (2011). Quantificação e análise do custo da sobra limpa em unidades de alimentação e nutrição de uma empresa de grande porte. Revista de Nutrição, 24, 593-604.

Souza, E. C., Leite, A. J. D., \& Costa, A. F. D. M. (2016). Avaliação dos fornecedores de gêneros alimentícios de um serviço de nutrição e dietética hospitalar em Maceió AL. Hig. aliment, 54-58.

Teixeira, F., Nunes, G., \& Antonovicz, S. (2017). Principais fatores associados aos índices de desperdício em Unidades de Alimentação e Nutrição: uma revisão integrativa. Saúde em Revista, 17(47), 42-50.

Teixeira, T., Milet, Z., Carvalho, J., Biscontini, T. M. (2007). Administração Aplicada às Unidades de Alimentação e NutriçãoEditora Atheneu. 